\title{
Gestures by Children and Adults on Touch Tables and Touch Walls in a Public Science Center
}

\author{
Lisa Anthony ${ }^{1}$, Kathryn A. Stofer ${ }^{2}$, Annie Luc ${ }^{1}$, Jacob O. Wobbrock ${ }^{3}$ \\ ${ }^{1}$ Dept. of CISE \\ University of Florida \\ Gainesville, FL 32611 USA \\ ${ }^{2}$ Dept. of Agricultural Education \\ and Communication \\ University of Florida \\ \{lanthony@cise.ufl.edu, \\ annieluc@ufl.edu\} \\ Gainesville, FL 32611 USA \\ ${ }^{3}$ The Information School
DUB Group
University of Washington
Seattle, WA 98195 USA
wobbrock@uw.edu
}

\begin{abstract}
Research on children's interactions with touchscreen devices has examined small and large screens and compared interaction to adults or among children of different ages. Little work has explicitly compared interaction on different platforms, however. Large touchscreen displays can be deployed flat, as in a table, or vertically, as on a wall. While these two form factors have been studied, it is not known what differences may exist between them. We present a study of visitors to a science museum, including children and their parents, who interacted with Google Earth on either a touch table or a touch wall. We compare the types of gestures and interactions attempted on each device and find several interesting results, including: users of all ages tend to make standard touchscreen gestures on both platforms, but children were more likely than adults to try new gestures. Users were more likely to perform two-handed, multi-touch gestures on the touch wall than on the touch table. Our findings will inform the design of future interactive applications for each platform.
\end{abstract}

\section{CCS Concepts}

- Human-centered computing Gestural input

- Human-centered computing Empirical studies in HCI

- Human-centered computing Touch screens

- Human-centered computing Field studies

- Human-centered computing Collaborative interaction

\section{Keywords}

Touchscreen interaction; touchscreen gestures; interactive tabletops; interactive wall displays; collaboration; child-computer interaction; public displays.

\section{INTRODUCTION}

Touchscreen interaction has experienced a sharp increase in availability and prevalence over the past decade, with the commercial availability and widespread adoption of touchscreen smartphones and tablet computers [1,20,30]. Large touchscreen devices such as tabletops and smart walls are also becoming more prevalent, in settings such as science museums and classrooms $[22,23]$. Research on children's interactions with touchscreen devices has examined both small and large screens [2$4,24,33,34,42,45,52]$, compared children's interaction to adults [2-

Permission to make digital or hard copies of all or part of this work for personal or classroom use is granted without fee provided that copies are not made or distributed for profit or commercial advantage and that copies bear this notice and the full citation on the first page. Copyrights for components of this work owned by others than ACM must be honored. Abstracting with credit is permitted. To copy otherwise, or republish, to post on servers or to redistribute to lists, requires prior specific permission and/or a fee. Request permissions from Permissions@acm.org. IDC '16, June 21-24, 2016, Manchester, United Kingdom. (C) 2016 ACM. ISBN 978-1-4503-4313-8/16/06...\$15.00. DOI: http://dx.doi.org/10.1145/2930674.2930682
$4,8,24,26,27]$ and compared the interaction of children of different ages to each other $[3,4,42]$.

Little work has explicitly compared interaction on different platforms, however (e.g., [29,43]). For example, large touchscreen displays can be deployed flat, as in a table, or vertically, as on a wall. While these two form factors have been studied separately, it is not known what interaction differences may exist between them. Properties of human ergonomics may have an effect on what interactions will be comfortable and natural to users on different platforms; for example, extending one's arm out horizontally for long periods of time leads to an uncomfortable heavy feeling known colloquially as "gorilla arm” [10]. Children especially may find different form factors feel more or less natural. For example, children may experience specific challenges in stretching to reach farther corners of displays mounted vertically or horizontally. If we could effectively characterize what differences exist between these two form factors (or possibly confirm that differences are minimal), we could provide clearer design recommendations on the deployment and interactions to use in either setting.

To this end, in this paper we present a study of visitors to a science museum, including children and their parents (Figure 1). In our study, visitors interacted with Google Earth on either a touch table or a touch wall at their own pace and with their own goals. For example, many visitors first tried to "find themselves" on the map by zooming into the city in which the museum was located. All visitor interactions were audio and video recorded. During our study, we recorded 2,456 gestures made by 66 visitors, of which 24 were children under about age 12 . We compare the types of gestures and interactions attempted on the touch table versus the touch wall, and by the children versus the adults.

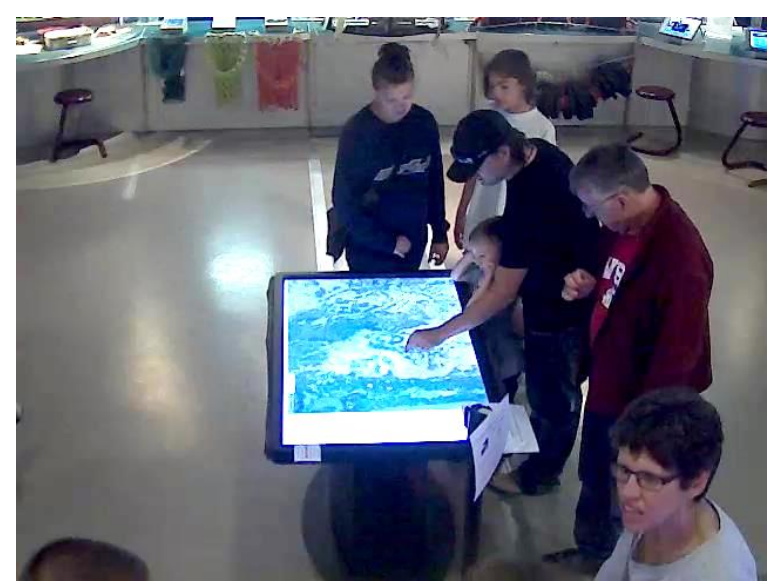

Figure 1. A multigenerational group interacts with Google Earth on a touch table at the science center and are recorded by the facility's remote camera system. 
In analyzing these gestures, we asked questions such as: What are the types and frequencies of different interactions for each platform? What are the affordances and constraints imposed and offered by each platform? How do interactions differ between children and adults? We labeled the gestures visitors made with details such as hand position, gesture type, and number of fingers in order to characterize the interactions and identify any challenges or opportunities for design. Our purpose was to determine what, if any, differences exist that may be exploited when designing interactive experiences for these displays.

We observed strong preference in users of all ages and on both platforms to make standard and familiar (e.g., smartphone) touchscreen gestures, but found that children were more likely than adults to try new gestures. Users were more likely to perform twohanded, multi-touch gestures on the vertical touch wall than on the touch table. We also saw consistent preference by users of all ages on both platforms for simple, one-finger, one-handed gestures in a vertical direction relative to the screen (e.g., "toward/away from me" on the table, "up/down" on the wall). Based on this analysis, we identify key design implications that should be considered when designing interactive touchscreen experiences on touch tables and touch walls for children and adults.

The contributions of this work include: (a) a deeper understanding of touch interaction differences and similarities on tabletops and wall displays; (b) a characterization of how children and adults differ when interacting with tabletops and wall displays; (c) a set of implications for the design of interactive experiences for touch tables and touch walls; and (d) a discussion of the challenges of this research methodology to aid future researchers in applying it to their own work. Our findings will inform the design of future interactive applications for touchscreen tabletop and touchscreen wall displays.

\section{RELATED WORK}

We survey related work on (1) understanding touchscreen interaction for tabletops and wall displays, especially for multigenerational groups; and (2) challenges of touchscreen interaction in informal science learning settings.

\subsection{Interactive Tabletops and Surfaces}

Interactive touch surfaces and displays have been an active area of research since the 1960s (e.g., see [40] for a survey of early systems). Early work in human-computer interaction investigations of interactive surfaces took an expert-first approach, designing gestures for people to use that were rooted in hardware, sensing, and algorithmic capabilities [53,55]. For example, Wu \& Balakrishnan [56] defined a set of 10 one- and two-handed gestures, including "tap," “double tap," "flick," "flat hand,” and "two corner hands" for use in a room planning application and reported challenges with recognizing these gestures on the DiamondTouch hardware [14] they used. Wilson et al. [53] incorporated a physics simulation into interactive tabletops to increase the realism of touch and gestural interactions, and supported new interactions such as "cupping" and "throwing / catching" that capitalized on the availability of physics. A notable exception to this system-first model is the work of Liu et al. [31] who studied how people rotated and translated pieces of paper on tabletop surfaces, and then designed new algorithmic techniques for detecting similar gestures.

More recently, a shift has occurred in research on interactive surfaces toward the concept of user-defined gestures [37,55]. Popularized by Wobbrock et al. [54,55], this concept focuses on the design of gestural interaction based on users' intuition. The methodology entails showing a user the effect of an as-yet- undefined gesture, and asking them to demonstrate a gesture that would make sense to them as the cause. Many follow-up studies have been conducted that use this method to elicit gestures from potential users for interactive surfaces for specific domains $[11,37,45]$. Morris et al. [37] compared gestures designed by expert gesture interaction researchers to those defined by novice users and found that users tended to prefer simpler gestures; also, the more people that independently proposed a gesture, the more likely it was to be rated highly by users. Cohé \& Hachet [11] explored userdefined 2D gestures to manipulate 3D objects on interactive surfaces. Rust et al. [45] specifically examined user-defined tabletop gestures for children, finding that prior touchscreen experience dominated both children's and adults' attempted gestures. Our study examines unprompted gestures performed by museum visitors to explore which gestures are used most by users for specific actions.

Complementary to the idea of user-defined gestures is the idea of observing user behavior interacting with tabletops and wall displays without prompting them for specific input actions [24,27,42,51]. John Tang [51] studied how people interacted with physical objects in a collaborative drawing scenario to inform the design of interactive tabletops based on existing behaviors. Hinrichs \& Carpendale [24] examined use of a touchscreen tabletop in a museum setting by 20 children and 20 adults, and found that people may use different gestures for the same action depending on age, context, and overall intention. Rick et al. [42] reported design considerations for group use of a tabletop in the classroom, such as equity of participation and use of touch space. Hornecker [27] recorded gestural interactions on an interactive table for five multigenerational groups visiting a natural history museum, and reported interaction difficulties encountered by the participants. This latter method of observing natural and unprompted user interaction behavior is what we used in our study. Our study goes beyond prior work by using unobtrusive methods to collect open-ended gesture interaction data from a large group of visitors of all ages.

Previous work on general touchscreen interactions with single users and users in groups has provided many design considerations to improve usability. Ichino et al. [29] performed a controlled laboratory exhibit to compare user responses to different display angles in museums; users rated tilted displays as superior in attracting attention and ease of use and interaction. Rogers et al. [43] reported from a user study that a horizontal display resulted in members switching roles more often and having a greater awareness of the other group members, while the vertical display hindered collaboration. Ryall et al. [46] performed an in-the-wild study to identify design considerations relating to interactive tables; they found that people can be hesitant to touch the table simultaneously and that initial single-finger input is common. While previous work has provided insight into designing more efficient and usable interfaces, we go further in this work by presenting a larger and more diverse dataset of users and comparing between two interactive displays, a touch wall and touch table. By doing so, we contribute additional design recommendations for museum exhibit interfaces that can in turn lead to better engagement for children and adults.

\subsection{Touchscreen Interactions in Museums}

Designing touchscreen interactions in science museums often focuses on interfaces intended to foster informal science learning. Designing for this context comes with challenges based on the physical context as well as on balancing user desires for interaction with measurable learning outcomes. The physical context of museums poses obstacles both for collecting user data to inform 
design and for designing exhibits for optimal use [17]. One challenge in collecting user data is that visitors are often hesitant to take standard tests or even participate in short interviews that may interrupt their experience [18]. Observing visitor behavior as they interact with exhibits may lead to changes in their typical behavior as visitors try to please the observer if visitors discover the researcher observing them [26,28]. Block et al. [8], for example, studied visitor engagement around multi-touch tabletop science exhibits using video study and shadowing and found that the research methodology affected user engagement. Unobtrusive observation alone is also difficult due to space constraints [18]. In our study, we present a large dataset of unprompted visitor interactions in front of both a touch wall and touch table collected using an unobtrusive observational study method. This methodology enabled us to minimize changes to normal user behavior and highlight natural user interactions.

The physical environment also constrains touchscreen exhibit design especially in terms of lighting and available space to coordinate between multiple people. For example, users of tabletop displays may encounter issues caused by non-visible segments of the display [6], territoriality and simultaneous control $[7,25,49]$, and competitive versus collaborative tasks [9]. Vertically-oriented touchscreen displays in real world contexts face problems including lighting or glare [40]. Unintended touches and mistaken interactions can be a challenge for multigenerational groups (e.g., $[2,3])$. We examine the physical constraints presented in our study to contribute to this body of knowledge and also provide suggestions to improving the environment for future work.

Designing touchscreen interactions for learning outcomes in a museum setting presents another set of challenges. The mere existence of technology in a learning environment does not mean it will necessarily be used effectively for the purposes or in the ways the designers intend [44]. In addition, interactive tabletop applications for learning have typically been designed for interaction outcomes, without concrete reference to specific learning theories or learning outcomes, although they may involve instructors and teachers in the design process $[41,47,50]$. Moreover, people may not always approach an exhibit at the initial state, and can instead begin engagement at a later stage in the application [8], which affects the way in which users perceive exhibits. Though we do not measure learning or engagement in our study, we believe that the design insights we provide from our observed user gestures and interactions can build a foundation for future work to study engagement and learning.

In addition to these challenges are the effects of social dynamics on group interaction. Crowley et al. [12] showed that joint parent-child interactions in a children's museum resulted in children having a longer, broader, and more focused exploration than when children interacted alone. Hinrichs et al. [25] presented EMDialog, an interactive presentation to examine the incentives for approaching an exhibit. They found that the display and visualization, as well as seeing other users interact on the exhibit, were both factors that affected group exploration. Marshall et al. [32] performed an inthe-wild study to determine how users began to engage with a tabletop interface and found that cohesive groups formed slowly, as in a buffet line, instead of a cohesive group of family or friends. Peltonen et al. [38] presented coded observations of user interactions with a large multi-touch display in a city center and found that the interface allowed for strangers to come into contact to interact together. Diamond [13] showed evidence that social

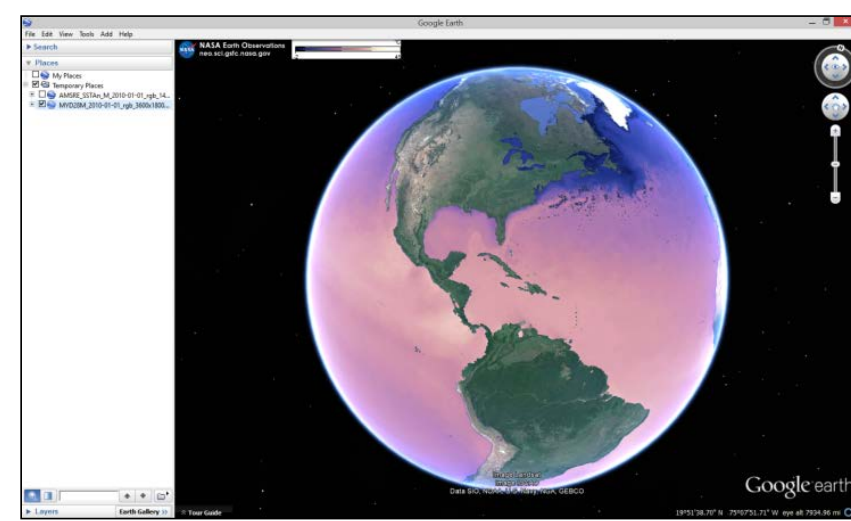

Figure 2. Screenshot of Google Earth interface used on the touch table and touch wall during our observation period.

interactions between visitors can stimulate learning at exhibits, and that teaching is a fundamental aspect of the interactions. Evans et al. [16] studied collaborative groups of adolescent students in live classrooms using interactive tabletops to accomplish group tasks. They found that various touch and gesture patterns were correlated with positive and negative collaborative behaviors, raising the possibility of automatic intervention. It is important to investigate the gestures made by users in groups to further inform better usability in museum contexts. We present findings of both individual and group interactions to better understand how to design interfaces to suit both types of audiences.

Finally, it is important to understand how to maximize usability to promote engagement and potentially informal learning. However, providing evidence of learning in informal environments remains challenging due to the highly personal nature of the experiences (e.g., [17,39]). Recent efforts to study tabletop interactions in museums focus on engagement as a proxy or initial condition for learning [28], measuring visitor interaction dwell time and talk $[22,23,26]$. However, the exhibit interactions designed in that work, by the authors' own admission, do not always support more openended exploration choices [26] or a variety of touchscreen gestures. We go beyond prior work by investigating what gestures museum visitors attempt in open-ended exploration, and examine how these afford or constrain general interaction. With more insights into how users interact best with large-scale interactive displays, we will be able to make progress on discovering how those gestures can lead to better engagement.

\section{METHOD}

Participants were self-selected visitors to a marine science center in the Pacific Northwest of the United States. Visitors often interacted in groups, typical of patterns of museum visitation [21]. Participants interacted with either a touch table exhibit or touch wall exhibit on three weekdays in July 2014. During observation time periods, the exhibits displayed Google Earth ${ }^{1}$, an interactive, web-based program displaying visible satellite imagery of the Earth. We selected this program because (a) it was already fully functional and would run on both the table and the wall, (b) it supported two-handed multi-touch interaction, and (c) it allowed for domain-relevant exploration of Earth's land and oceans. Options for adding layers such as roads and 3D buildings were turned off for this observation period. Visitors were allowed to choose layers of satellite ocean data as overlays, one showing average sea surface temperature and one showing anomalous sea

\footnotetext{
${ }^{1}$ http://www.google.com/earth
} 
surface temperature. However, few visitors were observed using these layers, so they will not be discussed further. Visitors were able to interact with the layer selection sidebar on the left-hand side of the screen, the main globe portion of the exhibit, or a navigation (zoom or pan) tool in the upper-right portion of the exhibit (Figure 2), hereafter "widget".

Visitor interactions with the exhibits were recorded using an unobtrusive camera system (see Figures 1, 3, 4, 6, 7, 9, and 10) mounted by the museum in select locations, similar to a closedcircuit security system. Cameras recorded continuously while the center was open. Ethics review of the use of this recording system was obtained under the jurisdiction of the marine science center; we additionally obtained ethics review approval to conduct our specific observations and interviews with the visitors.

The touch wall and touch table were the same 55" (diagonal) multitouch interactive screen manufactured by Ideum ${ }^{2}$, with the touch table (55" Pro) mounted as a stand-alone table in the middle of an exhibit hall (Figure 1) and the touch screen (55" Presenter) mounted on the wall of a different exhibit hall (Figure 7). Both capacitive touchscreen displays supported 60 simultaneous touch points, and were able to reject palm resting touches. The touch table was surrounded by interactive exhibits related to marine science research and live marine animals in tanks. The touch wall was in an exhibit hall with interactive pieces on the topic of remote sensing.

All visitors that interacted with the exhibit for more than one minute in the observation period were included in the analysis. Only four groups were excluded due to brief interaction times during our observations. Observation periods were defined as times during regular center open hours in which the researchers were present and Google Earth was running on the touch table or touch wall. Visitors were assigned to post-hoc visitor "groups" by the third author based on their joint interactions with the touchscreen exhibits.

We used the camera footage recorded of each exhibit during the observation period to label the gestures that were performed by every museum visitor that interacted with either the touch table or the touch wall. We recorded the following information:

- group member performing a gesture,

- approximate age group of the group member (adult, child, or teen),

- type of gesture (based on standard touchscreen gestures, e.g., swipe, tap, pinch, rotate, also “other”),

- hand(s) used in the gesture (which one, or both),

- finger(s) used in the gesture (per hand),

- exhibit part interacted with (globe or widget),

- direction of gesture (relative to the visitor position in front of the exhibit, e.g., up, down, left, right, clockwise, counterclockwise),

- hand position during the gesture (e.g., fingers splayed or tucked).

Some gestures in the video footage were partially or completely occluded due to camera angles or the presence of another visitor blocking the camera. We labeled as much of each gesture as was easily discernible and otherwise marked entries as "occluded." Overall, only $7 \%$ of the gestures performed during the observation period were completely occluded.

Because the labeling process consisted only of objective categories (other than perhaps the age group of the visitor), this process was treated as labeling for "ground truth" rather than qualitative coding. To calibrate the researchers for the labeling process, an initial batch of gesture labeling was performed by the first three authors on a

\begin{tabular}{|l|l|l|l|l|}
\hline & Adult & Child & Teen & Total \\
\hline Table & 573 & 467 & 29 & 1069 \\
& $(33,6 \%)$ & $(2,<1 \%)$ & $(5,17 \%)$ & $(40,4 \%)$ \\
\hline Wall & 534 & 368 & 485 & 1387 \\
& $(64,12 \%)$ & $(39,11 \%)$ & $(18,4 \%)$ & $(121,9 \%)$ \\
\hline Total & 1107 & 835 & 514 & 2456 \\
& $(97,9 \%)$ & $(41,5 \%)$ & $(23,4 \%)$ & $(161,7 \%)$ \\
\hline
\end{tabular}

Table 1. Number of gestures in the dataset separated by age and platform. $\mathbf{N}=\mathbf{2 , 4 5 6}$. In parentheses, the number of occluded gestures by age and platform.

subset of videos containing one group interaction of approximately the same duration for each exhibit (touch wall: 2 minutes (m) 48 seconds (s); touch table: $2 \mathrm{~m} 59 \mathrm{~s}$ ). Researchers first identified when a gesture occurred (e.g., start and stop times, defined as the time the user's hand touched the screen to the time the user's hand left the screen). After this initial labeling round, the authors met to discuss any difficult-to-discern (e.g., occluded) gestures.

Next, the first three authors labeled another subset of observations, which included one group from the touch table and one from the touch wall (both $1 \mathrm{~m} 56 \mathrm{~s}$ ). Again, the authors met to discuss difficult-to-see (occluded) gestures. Then, the authors agreed that understanding of the labeling scheme was sufficient and agreed upon heuristics for how to handle occluded gestures. At this point, the third author labeled all of the remaining videos. To validate the final labeling of the data, the first and second authors each further labeled a subset of the remaining videos that comprised approximately $10 \%$ of the total interaction time, 4 groups from the touch wall ( $5 \mathrm{~m} 22 \mathrm{~s}$ total) and 1 group from the touch table ( $3 \mathrm{~m}$ $42 \mathrm{~s}$ total). The first and second authors confirmed through this spot-check that overall labeling of gesture characteristics had been applied correctly and sufficiently.

\section{DATASET AND PARTICIPANTS}

During the observation periods, 37 visitors were observed in eight groups at the touch table exhibit, and 29 visitors were observed in 11 groups at the touch wall exhibit. Based on visual assessment, one researcher labeled each individual who interacted with the touch table or touch wall by gender and age; age was labeled as “adult” (roughly, 18 years and up), “teen” (roughly, 13 to 17), and "child” (roughly, 12 years and under). Overall, 37 visitors (56\%) were adults, 5 visitors (8\%) were teens, and 24 visitors (36\%) were children. Because of the small number of teenage users in our dataset, we do not include them explicitly in our analysis. Based on informal interviews conducted after visitors interacted with the exhibit, we estimate that about $60 \%$ of visitors to the site during our observation period were relatively familiar with the Google Earth program before interacting with the exhibit.

The average amount of time each group interacted with the exhibits was 4 minutes 38 seconds for the touch table (total: 37 minutes 2 seconds across eight groups) and 4 minutes 45 seconds for the touch wall (total: 52 minutes 20 seconds across 11 groups). Median time each group interacted with the exhibits was 3 minutes 16 seconds (table), and 1 minute 56 seconds (wall).

The total number of gestures we observed was 2,456; a breakdown by user age and exhibit platform is given in Table 1 . Since four groups were labeled prior to revising the labeling scheme to include the direction of the gesture and the hand posture, this information is missing for these four groups, accounting for 278 of the gestures (11\% overall).

\footnotetext{
${ }^{2}$ http://ideum.com/
} 


\begin{tabular}{|l|c|}
\hline \multicolumn{1}{|c|}{ Gesture Type } & $\begin{array}{c}\text { Number of Gestures } \\
\text { (\% of } \mathbf{2 , 4 5 6 )}\end{array}$ \\
\hline Swipe-straight & $846(34 \%)$ \\
\hline Pinch-in & $280(11 \%)$ \\
\hline Tap-multiple & $246(10 \%)$ \\
\hline Tap-single & $225(9 \%)$ \\
\hline Pinch-out & $211(9 \%)$ \\
\hline Swipe-arc & $141(6 \%)$ \\
\hline Other & $115(5 \%)$ \\
\hline Point-to-screen & $107(4 \%)$ \\
\hline Rotate & $89(4 \%)$ \\
\hline Above-the-surface & $35(1 \%)$ \\
\hline
\end{tabular}

Table 2. Gesture types observed. $N=2,456$ gestures. $7 \%$ of gestures were occluded and not counted here.

\section{FINDINGS}

We present patterns of interaction based on how gestures were performed on both platforms. Throughout the analyses, although we collected a total of 2,456 gestures, some gesture features were occluded and therefore the $N$ for each comparison may differ.

\subsection{Characterizing Gestures}

We labeled gesture type based on standard touchscreen gestures, and allowed gestures to be marked as "other" and separately annotated if the gesture did not fit into a predefined category. Table 2 shows the gesture types and their frequencies in our dataset. The distribution is significantly different from chance by a Chi-Square test $\left(\chi^{2} 9, \mathrm{~N}=2295=2077.5, \mathrm{p}<.0001\right)$.

The most commonly used gesture type differed based on what the user was intending to interact with $\left(\chi^{2} 8, \mathrm{~N}=2256=567.0, \mathrm{p}<.0001\right)$. When the user was interacting with the globe itself (87.5\% of the dataset), the most common gesture was "swipe-straight." When the user was interacting with on-screen Google Earth widgets such as the zoom bar or the layers selector, the most common gesture was "tap-multiple." Tap-multiple encompassed everything from two rapid back-to-back taps in the style of a mouse double-click to more than two repeated taps with a continuous rhythm.

Direction and rotation. Both swiping gestures and the rotate gesture have a gesture direction. Direction for the swiping gestures differs conceptually by platform, e.g., on the table: "toward me", "away from me”, "to the left”, "to the right”, and on the wall: "up”, "down”, "left", "right”. We treat the cardinal directions for each platform as conceptually equivalent, that is, we group "toward me" and "down" under the same label "south" and so on. Direction for the rotating gesture is the same for both platforms, that is, clockwise or counterclockwise. The most frequently appearing direction for

\begin{tabular}{|l|l|l|}
\hline $\begin{array}{l}\text { Numbers } \\
\text { of } \\
\text { Fingers }\end{array}$ & $\begin{array}{l}\text { No. of One-Handed } \\
\text { Gestures (\%) }\end{array}$ & $\begin{array}{l}\text { No. of Two-Handed } \\
\text { Gestures (\%) }\end{array}$ \\
\hline $\mathbf{1}$ & $1448(80 \%)$ & n/a \\
\hline $\mathbf{2}$ & $215(12 \%)$ & $299(78 \%)$ \\
\hline $\mathbf{3}$ & $19(1 \%)$ & $4(1 \%)$ \\
\hline $\mathbf{4}$ & $15(1 \%)$ & $11(3 \%)$ \\
\hline $\mathbf{5}$ & $105(6 \%)$ & $0(0 \%)$ \\
\hline $\mathbf{6}$ & n/a & $4(1 \%)$ \\
\hline $\mathbf{7}$ & n/a & $0(0 \%)$ \\
\hline $\mathbf{8}$ & n/a & $16(4 \%)$ \\
\hline $\mathbf{9}$ & n/a & $0(0 \%)$ \\
\hline $\mathbf{1 0}$ & n/a & $50(13 \%)$ \\
\hline
\end{tabular}

Table 3. Number of gestures, both single-handed and two-

handed, by numbers of fingers used. ( $N=1,993$ one-handed gestures and 415 two-handed gestures.) $2 \%$ of gestures were occluded in this category and not counted here.

these gestures is "toward me” / "down”, with 249 gestures (24\%; $N$ = 1,020 gestures). "Right” and "away from me” / "up” are roughly equally represented with 171 (17\%) and 163 (16\%), respectively. "Left" is infrequent, with 46 gestures (5\%). The distribution is significantly different from chance by a Chi-Square test $\left(\chi^{2} 9, \mathrm{~N}=1013\right.$ $=537.8, \mathrm{p}<.0001$ ). (Recall that direction is not available for four groups, corresponding to $11 \%$ of the gestures.)

Of the rotating gestures, counterclockwise rotating (to the left) was more frequently observed than clockwise rotating (to the right), with 145 gestures (14\%) and 90 gestures (9\%), respectively, a significant difference by a Chi-Square test $\left(\chi^{2}{ }_{1, N}=235=12.87, \mathrm{p}<\right.$ $.05)$. This difference could be due to most gestures being made with the right hand, discussed below, since rotating one's right-hand to the right is less ergonomic than vice versa. In the video stills sequence shown in Figure 3, the adult male rotates his right hand counterclockwise starting from anatomically semi-pronated and moving to a pronated position (e.g., rotated with palm down).

Single and multi-touch. Single touch interactions (one hand, one finger) far outweighed multi-touch interactions in our dataset, with 1,448 gestures $(66 \%)$ versus 741 gestures (34\%), a significant difference by a Chi-Square test $\left(\chi^{2} 1, \mathrm{~N}=2189=228.3, \mathrm{p}<.0001\right)$. Users only used both hands to interact with the globe itself, never to interact with onscreen widgets. Although we do not have handedness reports for all users in our dataset, for single-hand gestures, the right hand was used far more often to make gestures than the left hand, 1,554 gestures (78\%) versus 439 gestures (22\%), a significant difference by a Chi-Square test $\left(\chi^{2} 1, \mathrm{~N}=1993=623.8, \mathrm{p}<\right.$ .0001). (This pattern might be explained by the dominance of right-
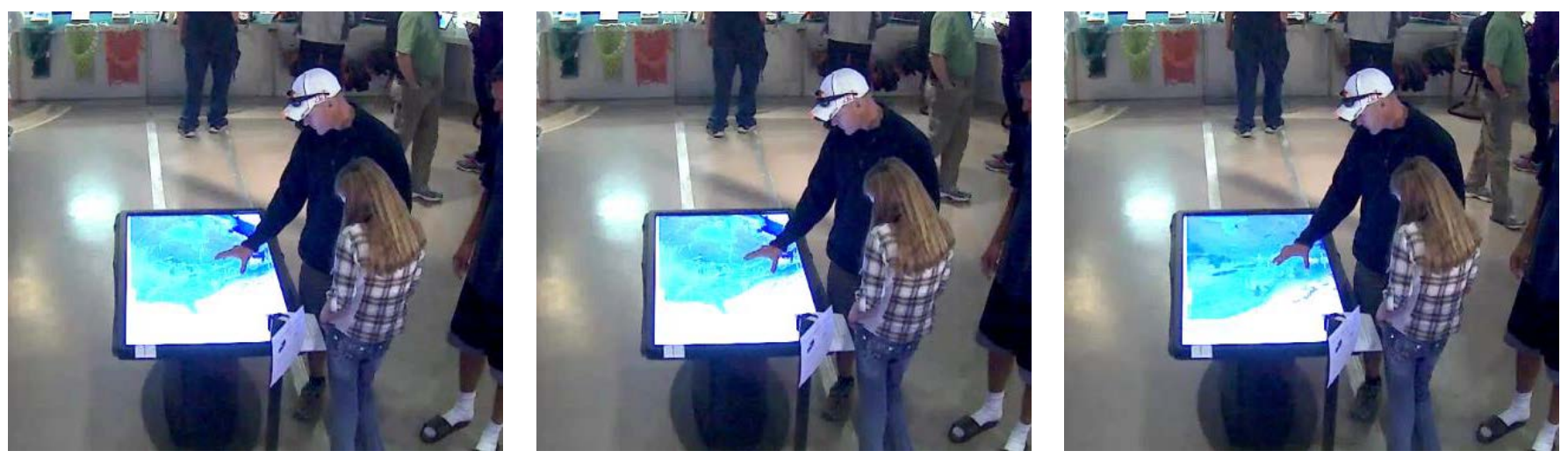

Figure 3. In this series of video stills, an adult male performs a counterclockwise rotate gesture at the touch table. Rotating counterclockwise was nearly twice as common as rotating clockwise. 


\begin{tabular}{|l|l|l|}
\hline $\begin{array}{l}\text { Individual } \\
\text { Fingers Used }\end{array}$ & $\begin{array}{l}\text { No. of One-Handed } \\
\text { Gestures (\%) }\end{array}$ & $\begin{array}{l}\text { No. of Two-Handed } \\
\text { Gestures (\%) }\end{array}$ \\
\hline Index & $1519(81 \%)$ & $336(86 \%)$ \\
\hline Middle & $477(25 \%)$ & $125(32 \%)$ \\
\hline Ring & $166(9 \%)$ & $72(18 \%)$ \\
\hline Pinky & $151(8 \%)$ & $71(18 \%)$ \\
\hline Thumb & $344(18 \%)$ & $70(18 \%)$ \\
\hline
\end{tabular}

Table 4. Number of gestures, one-handed and two-handed, performed using each individual finger. Gestures may include more than one finger. $(\mathrm{N}=1,877$ one-handed gestures and 390 two-handed gestures.) Use of individual fingers could not be determined for $8 \%$ of gestures.

handedness in the population [35]). Table 3 shows the distribution of number of contact points (e.g., fingers) with one-handed versus two-handed interaction in the dataset.

When comparing individual finger usage between hands, users more often used multiple fingers on the right hand than on the left (413 gestures, 21\%, versus 149 gestures, 17\%, respectively), a marginally significant difference by a Chi-Square test $\left(\chi^{2} 1, \mathrm{~N}=2688=\right.$ $3.395, \mathrm{p}=.0654)$. Users also exclusively used only a single finger to interact with the onscreen widget.

The most frequently used finger over all gestures regardless of hand, number of hands, number of fingers, age, platform, or what the users are interacting with is the index finger. Table 4 shows the distribution of individual fingers being utilized in one- and twohanded gestures, which is significantly different from chance by a Chi-Square test $\left(\chi^{2} 4, \mathrm{~N}=3331=41.9, \mathrm{p}<.0001\right)$.

We also noted that there are fewer combinations of fingers or individual fingers used on the left hand than the right hand $\left(\chi^{2} 5\right.$, $\mathrm{N}=2560=47.4, \mathrm{p}<.0001)$. This pattern might again be explainable by the dominance of right-handedness; perhaps users are more comfortable and dexterous with multiple fingers on their right hand than their left

Hand posture. We considered the posture of the user's hand and fingers during performance of each gesture, noting two main possibilities: "tucked," in which the user's unused fingers are tucked into the palm, and "splayed," in which the user's unused fingers are splayed out straight. In Figure 4, the adult male's hand is tucked as he points, while the child's hand is splayed as she manipulates the table. Hand posture information is not available for four groups due to the same reasons as for direction (revising the labeling scheme). Overall, gestures were performed more often in the splayed posture versus the tucked posture, 1208 gestures, $56 \%$, and 899 gestures, $42 \%$, respectively $\left(\chi_{1, \mathrm{~N}=2107}^{2}=45.3, \mathrm{p}<.0001\right)$.

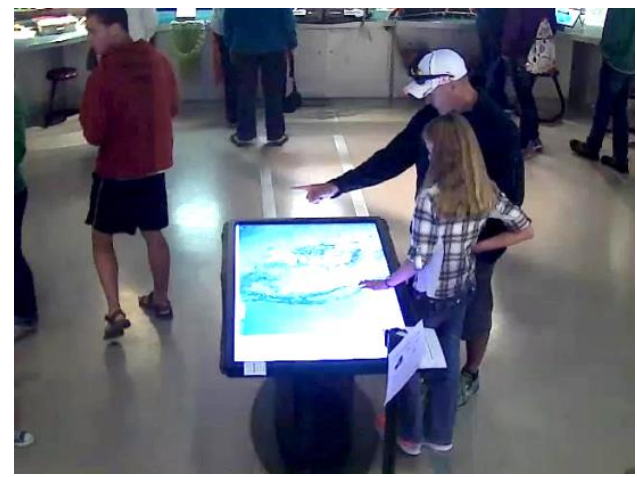

Figure 4. In this video still, an adult male points to the touch table with his hand tucked; the child performs a "swipe-straight" gesture while her hand is splayed.

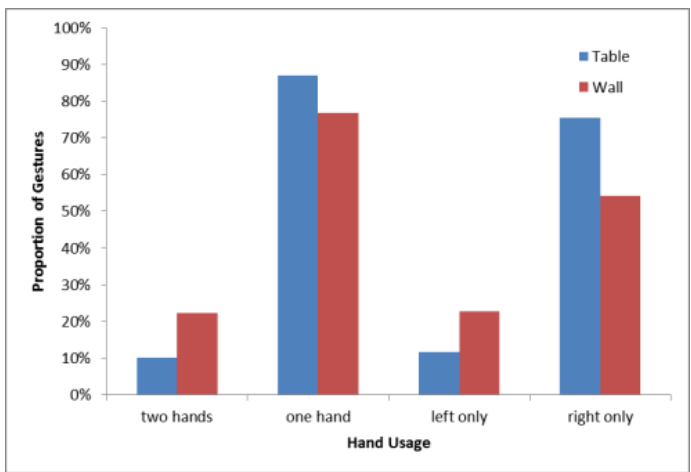

Figure 5. Proportion of gestures performed with two hands, one hand, and each individual hand on both the touch table and the touch wall.

We also noted trends in differences in frequency of hand posture based on the gesture being performed (and did not perform statistical tests since they were post-hoc). The top three gestures with majority splayed hand postures were "pinch-in" (71\% vs. $29 \%$ ), "swipe-arc" (67\% vs. 32\%), and "other" (63\% vs. $37 \%)$. The top two gestures with tucked hand postures were "point to screen" ( $85 \%$ vs. $13 \%$ ), and "tap-single" (50\% vs. $48 \%$ ); all other gesture types were performed splayed the majority of the time.

\subsection{Differences by Platform}

We compared interaction patterns between the two platforms. The distribution of gesture types performed by the users was roughly equivalent when we consider gestures performed on the touch table versus the touch wall but still different than chance $\left(\chi^{2}{ }_{5, N=1769}=\right.$ 25.6, $\mathrm{p}<.0001)$. We observed a post-hoc trend that users were twice as likely to perform the "swipe-arc" gesture on the touch wall as compared to the touch table. This pattern could be due to ergonomic affordances of moving one's arm through the air versus on a surface in an arcing motion. Further analysis based on properties of human kinesthetic motion may reveal the underlying cause of this type of pattern.

Single and multi-touch. Visitors of all ages used both hands to interact with the wall almost twice as frequently as they used both hands to interact with the table (Figure 5), a significant difference by a Chi-Square test $\left(\chi^{2} 1, \mathrm{~N}=2408=60.8, \mathrm{p}<.0001\right)$. Also, users were more likely to use their right hand on the touch table, and their left hand on the touch wall $\left(\chi^{2} 1, \mathrm{~N}=1993=74.4, \mathrm{p}<.0001\right)$. Although there are potential confounds such as handedness, one plausible reason for this behavior is the greater degree of flexibility on the touch table- - users can walk around the table to find a comfortable place

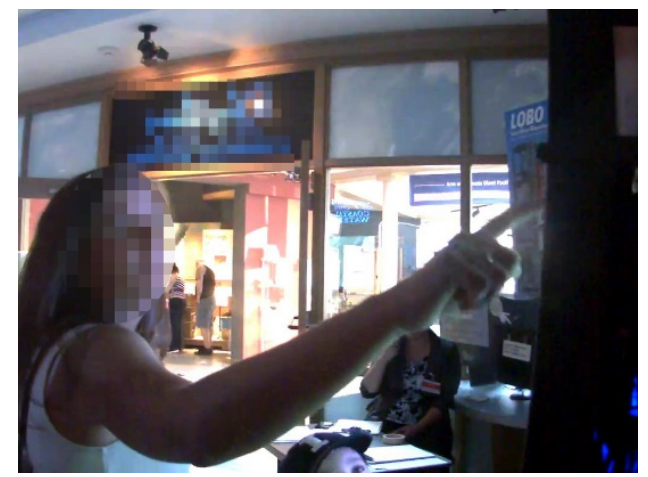

Figure 6. In this video still, an adult female interacts with the touch wall with fingers tucked; this hand posture was more frequent on the touch wall than the touch table. 


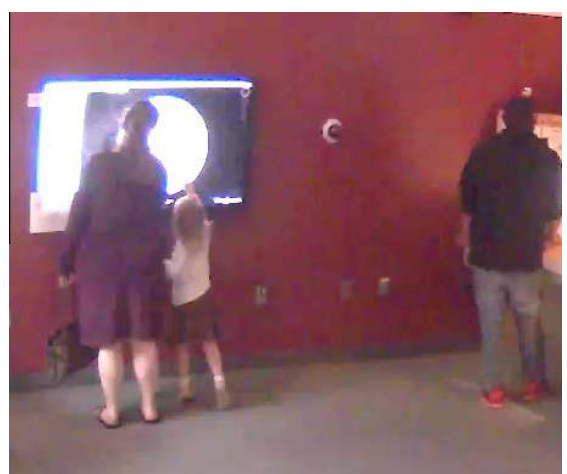

Figure 7. In this video still, a child stands on tip-toes next to an adult woman but can't reach the top half of the display.

to use their right hand, whereas at the wall, there are fewer positions possible.

Hand posture. On the touch table, a splayed hand posture is significantly more common, at least for adults and children, while the opposite is true on the touch wall $\left(\chi^{2} 1, \mathrm{~N}=2107=5.96, \mathrm{p}<.05\right)$. This interaction pattern is true for the most part regardless of whether the users are interacting with the globe or the widget. However, we also noted a post-hoc trend that adults in our dataset performed gestures on the touch wall with hand postures much more equally divided between splayed and tucked when interacting with the globe itself, but while interacting with the onscreen widgets on the touch wall, adults tend to have their hand tucked, as shown in Figure 6.

\subsection{Differences by User Age}

The gestures in our dataset were performed by users frequently interacting in multigenerational groups composed of adults, children, and teens. Due to the low prevalence of teen visitors in our dataset, we focus on contrasting children's gestures and adults' gestures.

The distribution of the top 5 gesture types (Table 2) did not differ between children and adults but were significantly different from chance $\left(\chi^{2}\right.$, $\left.N=1769=36.3, p<.0001\right)$. However, children tended to perform "Other" types of gestures more often than adults, especially on the touch table ( $11 \%$ vs. 3\%, respectively), a significant difference by a Chi-Square test $\left(\chi^{2}{ }_{1, N}=1769=9.49, \mathrm{p}<\right.$ $.01)$. The use of specialized gestures is discussed in more detail later. In addition, a post-hoc trend showed that children tended to use "pinch-out" more frequently on the touch wall than did adults (14\% vs. $7 \%$, respectively). When comparing the types of gestures tried by users of different ages, all standard touchscreen gestures were used by both adults and children on both platforms.

In terms of considering what parts of the interface the users tried to interact with, post-hoc trends showed that children virtually never used the onscreen widgets on the touch wall, as compared to adults. This pattern seems most likely due to the inaccessibility of the onscreen widgets in Google Earth, which were located in the upper right and left corners. When running on a touch wall, these widgets were too high up for children to reach, even when standing on tiptoes as shown in Figure 7. This type of occurrence highlights the challenges, and the importance, of doing this kind of work in a realworld context: this was the set-up at the museum and was the type of interactive experience available to children when they visited.

Direction and rotation. Adults performed significantly more circular (e.g., clockwise and counter-clockwise) gestures than children did $\left(\chi^{2} 3, \mathrm{~N}=760=13.1, \mathrm{p}<.0001\right)$, shown in Figure 8. In general, most users make more vertical (e.g., "toward" / "away" /

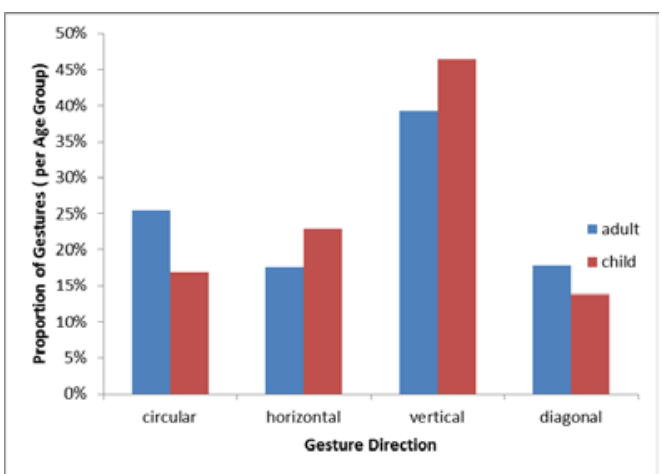

Figure 8. Proportion of gestures performed in each direction by children and adults. Note direction only applies for "swipe-straight", “swipe-arc”, and "rotate."

“up” / “down”) gestures than horizontal (“right” / "left”) gestures on both platforms.

As mentioned earlier, in rotate gestures, users tend to rotate in a counterclockwise direction (to the left). Examining this behavior by user age group shows a difference between children and adults: children seem more equally likely to rotate to the right or to the left, although this difference is not significant $\left(\chi^{2} 1, \mathrm{~N}=165=0.186\right.$, n.s. $)$. This interaction trend may be due to increased flexibility for children, although how development of fine motor control and manual dexterity may interact with this effect is not clear.

Single and multi-touch. Children are significantly more likely than adults to use more than one finger to perform their gestures with either hand, making two- and five-finger gestures more often than adults $\left(\chi^{2} 1, \mathrm{~N}=2105=25.3, \mathrm{p}<.0001\right)$. When considering which individual fingers are used in gestures, adults tend to use their middle finger nearly three times as often as children, a significant difference by a Chi-Square test $\left(\chi^{2} 5, \mathrm{~N}=2560=181.0, \mathrm{p}<.0001\right)$. In Figure 9 , the adult uses his middle finger to tap the widget. By the same test, adults are also more likely than children to use their middle finger in one-finger gestures ( $20 \%$ vs. $2 \%$ for adults and children, respectively). Furthermore, children are more likely to use all five fingers. In Figure 10, both children use all fingers on their right hand to manipulate the table.

We also observed some general post-hoc trends when considering more specific cases such as combinations of fingers per hand. When using two fingers on the left hand, children are more likely to use their thumb and index finger ( $8 \%$ vs. $1 \%$ for adults). When using two fingers on the right hand, using the index and thumb is the most common across both ages (adults: $5 \%$, children: $7 \%$ ), though adults

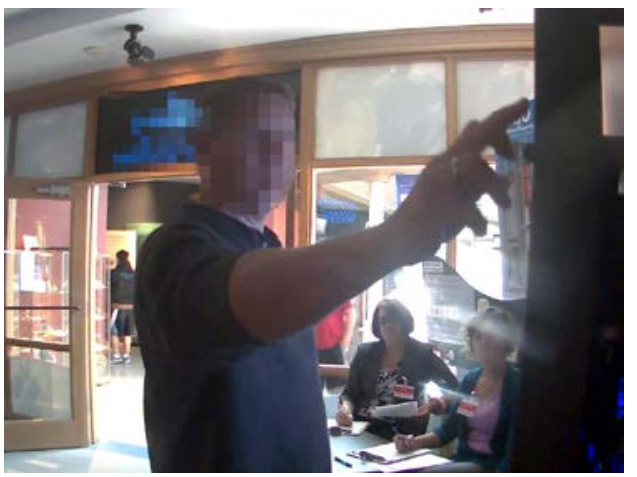

Figure 9. In this video still, an adult male uses his middle finger on the touch wall. Use of the middle finger alone to perform gestures was more common in adults than children. 
also use middle and thumb (6\%) equally frequently. Considering which combinations of fingers are likely to be used can inform the design of specialized multi-touch interactions.

\subsection{Specialized Gestures}

Although most of the gestures that visitors we observed were standardized touchscreen gestures like swipe, pinch, and rotate, other types of gestures that did not fit into these categories were also performed by some visitors. In all, 115 gestures (4.7\% of the total dataset) were labeled as "other."

Comparing just prevalence of standard to non-standard / "other" gestures, children used "other" gestures significantly more frequently than adults overall $\left(\chi^{2} 1, \mathrm{~N}=1769=9.49, \mathrm{p}<.01\right)$. Frequency of use of "other" gestures also differed significantly by platform $\left(\chi^{2} 1, \mathrm{~N}=2260=8.02, \mathrm{p}<.01\right)$. Children used "other" gestures approximately $11 \%$ of the time when interacting with the wall, whereas adults on both platforms and children interacting with the table used "other" gestures no more than $4 \%$ of the time. Potential explanations for the lower frequency of use of non-standard gestures by adults could be: (a) adults are more aware of standard gestures that are more likely to produce a satisfying result, (b) adults have a desire to "do museum" [28] correctly and "solve" or "complete" the activity in a prescribed manner, or (c) adults are more goal-oriented in their behavior than are children, who are more comfortable freely exploring.

The frequency of using "other" gestures differed significantly by visitor age group when comparing between what interface element visitors were interacting with (globe versus widget, $\chi^{2}{ }_{1, \mathrm{~N}=2256}=$ 48.8, $\mathrm{p}<.0001$ ). More specifically, adults most frequently used "other" gestures when interacting with the widget on the wall (16\%) compared to interacting with the globe on the wall or with either interface element on the table. Children, however, used other gestures on the table approximately equally as often when interacting with the globe (9\%) as with the widget (14\%). This pattern may be due to adults' increased familiarity with Google Earth, or increased familiarity with the standard gestures more likely to produce a desired result with interactive widgets.

We annotated the specific execution form of each of the "other" gestures and briefly describe some of the most common types here. Because there are so few occurrences of each type, we do not report statistical contrasts analyzing the frequency of these individual gesture types. Table 5 lists the top five "other" gesture types we observed in our dataset. Holding a finger or fingers on the screen without lifting them or moving them (e.g., "tap and hold") was the most frequently performed "other" gesture, used by adults in at least $38 \%$ of the "other" gestures on the table and $65 \%$ on the wall.

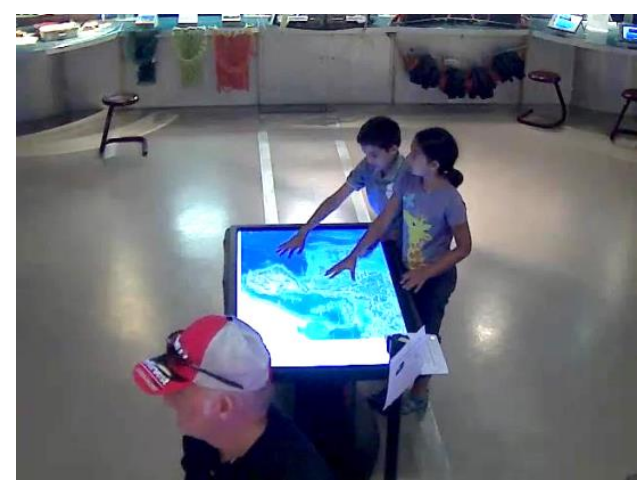

Figure 10. In this video still, two children interact together at the touch table and are using all five fingers to make "pinchin" and "swipe-straight" gestures.

\begin{tabular}{|l|l|}
\hline Non-Standard Gesture Types (name and short description) \\
\hline Tap and hold & $\begin{array}{l}\text { Touches the screen with one or more fingers } \\
\text { one or more times and holds contact for } \\
\text { more than a few seconds }\end{array}$ \\
\hline Tap and swipe & $\begin{array}{l}\text { Touches the screen with one or more fingers } \\
\text { then immediately swipes in a direction }\end{array}$ \\
\hline Circle(s) & $\begin{array}{l}\text { Touches screen and moves hand or fingers } \\
\text { in one or more repeated circular motions }\end{array}$ \\
\hline Pinch and tap & $\begin{array}{l}\text { Pinch in gesture with one hand while } \\
\text { tapping one or more times with the other } \\
\text { hand }\end{array}$ \\
\hline $\begin{array}{l}\text { Two-finger } \\
\text { rotate }\end{array}$ & $\begin{array}{l}\text { Holding two fingers on the screen and } \\
\text { making a circular motion }\end{array}$ \\
\hline
\end{tabular}

Table 5. Annotation of the top five "other" (non-standard) gesture types we observed in our dataset. The remaining non-standard gestures were extremely rare.

Children used some sort of stationary gesture far more frequently on the table ( $68 \%$ of "other" gestures) than the wall (17\% of "other" gestures). Variations of these stationary touches often involved holds following single or multiple taps. Additionally, when interacting on the wall, both adults (17\%) and children (33\%) used "other" gestures that were combinations of taps followed by swipes without lifting the hand. Both the tap-then-hold, and especially the tap-then-swipe, are reminiscent of "combo moves" from video games, and are becoming more common on mobile devices ("long tap"). Children frequently held their finger stationary on the screen without preceding it or following it with another gesture (17\%).

Regardless of platform, adults' “other” gestures were either hold or tap-then-hold combinations on the widget (100\% table, 100\% wall) more often than on the globe (23\% table, $43 \%$ wall). This behavior is likely due to the "button"-style interaction afforded by the widget; in the desktop version of Google Earth software, the widget can be manipulated with either a single-click or a click-then-hold of the mouse. Children used the hold or tap-then-hold touches with roughly equal frequency between the table (63\% globe, 93\% widget) and the wall (17\% of "other" interactions with the globe, but children had no "other" interactions with the widget on the wall). On the other hand, tap-then-swipe combinations were used more frequently when interacting on the wall with the globe than with the widget, for both adults (43\% vs. $0 \%$ ) and children (33\% vs. $0 \%$ ). Finally, repeated circling motions with a single finger or whole hands were used by two visitors as "other" gestures.

\section{DISCUSSION}

In general, we observed that visitors tend to make mostly standard touchscreen gestures in their interactions with both the touch table and touch wall. The distribution of which gestures were most frequently used did not differ by user age or by platform; the top three gestures were standard touchscreen gestures "swipe-straight", "pinch-in", and "tap-multiple". Based on our demographic data, we can extrapolate that touchscreen experience with smaller-screen devices outside of the museum setting dominates users' intuitions about gestures to perform and actions to attempt. Children tended to try "other" types of gestures more frequently than adults (about $11 \%$ of their gestures), which could be a product of less overall touchscreen experience or an attitude of exploration, or both. We found it interesting that the predominant mode of interaction was one-handed, in spite of the affordances of the large screens and support for multi-touch. Although still a minority of gestures overall, the use of two hands on the touch wall was twice that on the touch table (approximately $20 \%$ vs. 10\%). We observed consistent preference by users of all ages on both platforms for use 
of simple, one-finger, one-handed gestures that favor vertical gestures (e.g., toward/away from me on the table, up/down on the wall). These results highlight an emphasis on the user's own orientation toward the platform having a strong impact on perceptions of directionality and interactive degrees of freedom which affect the gestures they attempt.

\subsection{Design Implications}

We highlight design implications for gestural interaction most important to support on touch tables and touch walls, especially in the context of public displays in science museums. In contrast to previous work on touchscreen interaction in public settings that has focused on the social context and how it affects interaction [13,24,27,32,38,43], our design implications are motivated from a perspective of affording deeper engagement with the content.

Provide full support for standard touchscreen gestures. Users show a strong reliance on standard touchscreen gestures, and expect interactive touchscreens to respond to these gestures. Lack of response may cause frustration, leading the visitors to walk away from the exhibit without engaging more deeply with the content. We recommend all standard touchscreen gestures be supported with predictable results where possible.

Consider use of "lesser fitting" gestures for new features. If it does not make sense for an application to support "pinch-in" to produce a zoom action, perhaps the "pinch-in" gesture can be repurposed for another conceptually relevant action. This design feature would prevent discouragement by ensuring the application remains responsive no matter what the user tries, and might even encourage exploration and increased engagement.

Allow simple gestures to result in big actions. Users show strong preferences for simple, one-handed gestures. Design of effective touchscreen interactions for these settings will ensure that these simple gestures allow users to "dig in" deeply to the content and get engaged in the science inquiry more quickly. Use of more complex gestures for complex manipulations of onscreen data or content would keep these features hidden from most users.

Favor vertical interactions over horizontal. When considering the degrees of freedom to be supported by an application, the tendency of designers is to emphasize horizontal panning and swiping, possibly due to the landscape format of the display and legacy design of interfaces with top-of-screen menus and so on. In our study, however, users heavily favored vertical panning and swiping, possibly due to recent emphasis on such scrolling interactions on mobile touchscreens. Thus, we encourage designers to consider adopting this model in new interfaces for touchscreen tables and walls instead. Menus, if they are needed, can be pulled in from the left or right sides to allow for vertical swiping.

Consider children's tendency to explore and try unexpected gestures. By ensuring responsiveness is maintained, even in the face of unexpected gestures, interfaces could capitalize on this tendency to encourage and cultivate exploration. Designers should consider ways to guide children to make more anticipated gestures, or better, to allow the system to respond in constructive ways to unexpected gestures. Related to considering children's particular needs, children will not have the same reach as adults: laying out the interactive exhibits so that it is possible to access all functionality (e.g., not too high on the wall, not too centered on the table) will be important.

Treat multi-touch and two-handed gestures similarly to singletouch and one-handed gestures. One notable exception to users' preference for simple gestures is that on the touch wall, they were much more likely to use two-handed gestures engaging all fingers than on the touch table. However, the actual gestures used did not differ (e.g., swipe, pinch). This pattern could be due to different ergonomic affordances between the two platforms. This result complements prior work that found people utilize multiple fingers on interactive tabletops even where one finger would do [55]. Supporting seamless equivalence between multi-touch or twohanded gestures and single-touch or one-handed gestures with the same surface execution pattern (e.g., a one-handed swipe should result in the same action as a two-handed swipe), at least on the touch wall, will improve cross-platform gestural interaction.

\subsection{Future Applications}

We envision the impact of this work in part will be to inform the design of future touchscreen interfaces for exhibits in public settings such as science museums. A key aim of science museums and centers is to provide space for informal (e.g., out-of-school) science learning and exploration, through both physical and digital hands-on interactive exhibits. In the United States, informal or freechoice science learning institutions such as science museums and centers attract more visitors each year than the four major professional sports leagues combined [17]. Recently, researchers have begun to show that the experiences in these settings can produce more than a simple "spark of curiosity" (e.g., [15,21,39]), but there is still much work to be done to learn how to create compelling, rich experiences on interactive surfaces in these spaces to support robust learning. Our study is the foundation of a project in which human-computer interaction specialists (especially children's touchscreen interactions) are collaborating with learning science specialists (especially informal public science learning) to design, develop, and evaluate new experiences and generate design guidelines for these experiences. Initial directions include designing exhibits that react meaningfully to unexpected input and exploring the link between gestures and learning.

\subsection{Reflections on the Study Method and Limitations of the Work}

In the work presented in this paper, we faced several limitations to address in future studies. First, we had a very small number of teens in our dataset. This group is typically not a large user of science centers $[5,48]$, so it may remain a challenge to obtain data from teens without recruiting participants specifically. Also, museum visitors overall are not an ethnically diverse group [19], and our study population was no exception. The use of a remote camera system that could perform facial recognition and automatically estimate age or ethnicity would aid in focusing data collection on a more diverse subset of visitors.

There were also limitations with the set-up of the exhibits. We used off-the-shelf Google Earth software, which could not respond to all possible gestures beyond the standard ones. This limitation could affect frequency of observation of certain gestures, particularly non-standard gestures. Also, as users may have been familiar with the software, they may have tried fewer types of gestures. Where the visitors could stand in relation to the exhibits might have impacted the direction of their gestures and which hand they used at the touch table versus the touch wall differently. The camera angles limited our view for capturing some details, especially for the touch wall and especially for children, resulting in a number of occluded gestures affecting distributions by platform.

This study has characterized a wide dataset of gestures done by both children and adults on two large touchscreen displays. Future work could consider any impact of the display format (e.g., table vs. wall) on cognitive aspects of the interaction, especially in the context of learning at science centers. Although we did collect some 
demographic data in follow-up interviews with the participants, we did not ask children why they tried certain gestures in certain contexts. We plan to investigate this as we continue this research. In addition, future research could use a deductive approach to test whether the interactions of a new set of museum visitors follow similar patterns as were observed in this inductive study.

Finally, an ongoing challenge in research with public audiences in museums is designation of groups. Individuals who arrive in groups of family or friends do not visit all exhibit pieces or even entire exhibit halls in the same order or at the same time [36]. Groups break apart and re-form, so when more than one individual approaches and interacts with an exhibit piece at the same time, it is difficult to know for sure whether these de-facto groups match visitor perceptions of social ties. We can only make our best judgments based on our knowledge of museum audiences and other data such as recordings of the group entering or exiting the science center. Our decision in this study to identify a particular set of 15 visitors as a single group because their interaction times overlapped might have affected the average and median group interaction length for the touch table.

\section{CONCLUSION}

We presented an analysis of 2,456 gestures performed on either a touch table or a touch wall by 66 visitors ( 24 children) to a marine science center. We labeled the gestures to characterize interaction patterns and compared them across platforms and between children and adults. We observed strong preferences among all visitors on both platforms to use standard touchscreen gestures but found that children were more likely than adults to try new gestures. Users were more likely to perform two-handed, multi-touch gestures on the touch wall than on the touch table. Our study expands prior work observing gesture interactions of museum visitors $[8,24$ $27,29,32]$ to compare two touch platforms and to use unobtrusive methods to collect gesture interaction data. Based on our analysis, we present design implications for gestural interaction on large touchscreen displays, especially in public settings. The results of this work will form the foundation of next-generation experiences on interactive surfaces in these spaces.

\section{SELECTION AND PARTICIPATION OF CHILDREN}

Overall, 24 children participated in this research and were a selfselected sample based on their attendance at the science center. The science center is free to visit, with a suggested donation of \$5 per person. The remote camera system in the science center was approved by the governing ethics review body, and our specific research including use of the cameras, in-person observations, and follow-up interviews, was approved by our institution's ethics review body. Parents were approached for consent to speak with them and their children after they had interacted with the exhibit.

\section{ACKNOWLEDGMENTS}

The authors thank the Cyberlab project under direction of Dr. Shawn Rowe and travel support from NSF grant \#DRL-1114741.

\section{REFERENCES}

1. ABI Research. 2008. Touch Screens in Mobile Devices to Deliver \$5 Billion Next Year. FierceWireless. Retrieved January 24, 2016 from http://www.fiercewireless.com/pressreleases/touch-screens-mobile-devices-deliver-5-billion-nextyear

2. Lisa Anthony, Quincy Brown, Jaye Nias, Berthel Tate, and Shreya Mohan. 2012. Interaction and Recognition Challenges in Interpreting Children's Touch and Gesture Input on Mobile Devices. Proceedings of the ACM International Conference on Interactive Tabletops and Surfaces, ACM Press, 225-234. http://doi.org/10.1145/2396636.2396671

3. Lisa Anthony, Quincy Brown, Jaye Nias, and Berthel Tate. 2013. Examining the need for visual feedback during gesture interaction on mobile touchscreen devices for kids.

Proceedings of the International Conference on Interaction Design and Children, ACM Press, 157-164. http://doi.org/10.1145/2485760.2485775

4. Lisa Anthony, Quincy Brown, Berthel Tate, Jaye Nias, Robin Brewer, and Germaine Irwin. 2014. Designing smarter touchbased interfaces for educational contexts. Personal and Ubiquitous Computing 18, 6, 1471-1483. http://doi.org/10.1007/s00779-013-0749-9

5. Deanna Banks Beane. 2000. Museums and Healthy Adolescent Development: What We Are Learning from Research and Practice. Journal of Museum Education 25, 3, 3-8. http://doi.org/10.1080/10598650.2000.11510424

6. Hrvoje Benko, Andrew D. Wilson, and Ravin Balakrishnan. 2008. Sphere: multi-touch interactions on a spherical display. Proceedings of the ACM Symposium on User Interface Software and Technology, ACM Press, 77-86. http://doi.org/10.1145/1449715.1449729

7. Hrvoje Benko. 2009. Beyond flat surface computing: challenges of depth-aware and curved interfaces. Proceedings of the ACM International Conference on Multimedia, ACM Press, 935-944. http://doi.org/10.1145/1631272.1631462

8. Florian Block, James Hammerman, Michael Horn, Amy Spiegel, Jonathan Christiansen, Brenda Phillips, Judy Diamond, E. Margaret Evans, and Chia Shen. 2015. Fluid Grouping: Quantifying Group Engagement around Interactive Tabletop Exhibits in the Wild. Proceedings of the ACM SIGCHI Conference on Human Factors in Computing Systems, ACM Press, 867-876. http://doi.org/10.1145/2702123.2702231

9. John Bolton, Kibum Kim, Roel Vertegaal, and K L Canada. 2012. A comparison of competitive and cooperative task performance using spherical and flat displays. Proceedings of the ACM Conference on Computer Supported Cooperative Work, ACM Press, 529-538. http://doi.org/10.1145/2145204.2145286

10. Tim Carmody. 2010. Why “Gorilla Arm Syndrome” Rules Out Multitouch Notebook Displays. Wired. Retrieved January 24, 2016 from http://www.wired.com/2010/10/gorilla-armmultitouch/

11. Aurélie Cohé and Martin Hachet. 2012. Understanding user gestures for manipulating 3D objects from touchscreen inputs. Proceedings of Graphics Interface, Canadian Information Processing Society, 157-164.

12. Kevin Crowley, Maureen A. Callanan, Jennifer L. Jipson, Jodi Galco, Karen Topping, and Jeff Shrager. 2000. Shared Scientific Thinking in Everyday Parent-Child Activity. Science Education 85, 6, 712-732. http://doi.org/10.1002/sce.1035

13. Judy Diamond. 1986. The Behavior of Family Groups in Science Museums. Curator: The Museum Journal 29, 2, 139- 
154. http://doi.org/10.1111/j.2151-6952.1986.tb01434.x

14. Paul Dietz and Darren Leigh. 2001. DiamondTouch: a multiuser touch technology. Proceedings of the ACM Symposium on User Interface Software and Technology, 219-226. http://doi.org/10.1145/502348.502389

15. Kirsten M. Ellenbogen, Jessica J. Luke, and Lynn D. Dierking. 2007. Family learning in museums: Perspectives on a decade of research. In In principle, in practice: Museums as learning institutions, John H. Falk, Lynn D. Dierking and S. Foutz (eds.). AltaMira Press, 17-30.

16. Abigail C. Evans, Jacob O. Wobbrock, and Katie Davis. 2016. Modeling Collaboration Patterns on an Interactive Tabletop in a Classroom Setting. Proceedings of the ACM Conference on Computer-Supported Cooperative Work \& Social Computing, ACM Press, 858-869.

http://doi.org/10.1145/2818048.2819972

17. John H. Falk and Lynn D. Dierking. 2000. Learning From Museums: Visitor Experiences and the Making of Meaning. AltaMira Press, Plymouth, UK.

18. John Falk and Martin Storksdieck. 2005. Using the contextual model of learning to understand visitor learning from a science center exhibition. Science Education 89, 5, 744-778. http://doi.org/10.1002/sce.20078

19. Betty Farrell and Maria Medvedeva. 2010. Demographic Transformation and the Future of Museums. Washington, DC, USA. Retrieved January 24, 2016 from http://www.aamus.org/docs/center-for-the-future-ofmuseums/demotransaam2010.pdf?sfvrsn=0

20. W. David Gardner. 2008. iPhone: Catalyst For Touchscreen Revolution. InformationWeek. Retrieved January 24, 2016 from http://www.informationweek.com/mobile/mobiledevices/iphone-catalyst-for-touchscreen-revolution/d/did/1070343?

21. Joshua P. Gutwill and Sue Allen. 2009. Facilitating family group inquiry at science museum exhibits. Science Education 94, 4, 710-742. http://doi.org/10.1002/sce.20387

22. Kate Haley Goldman and Jessica Gonzalez. 2014. Open Exhibits Multitouch Table Use Findings. Retrieved January 24, 2016 from http://openexhibits.org/paper/oe-multitouchtable-use-findings/

23. Kate Haley Goldman and Jessica Gonzalez. 2014. Comparison of Multitouch Wall and Touch Table Format. Retrieved January 24, 2016 from http://openexhibits.org/paper/comparison-multitouch-walltouch-table-format/

24. Uta Hinrichs and Sheelagh Carpendale. 2011. Gestures in the wild: studying multi-touch gesture sequences on interactive tabletop exhibits. Proceedings of the ACM SIGCHI Conference on Human Factors in Computing Systems, ACM Press, 3023-3032. http://doi.org/10.1145/1978942.1979391

25. Uta Hinrichs, Holly Schmidt, and Sheelagh Carpendale. 2008. EMDialog: bringing information visualization into the museum. IEEE Transactions on Visualization and Computer Graphics 14, 6, 1181-8. http://doi.org/10.1109/TVCG.2008.127

26. Michael S Horn, Zeina Atrash Leong, Florian Block, Judy
Diamond, E. Margaret Evans, Brenda Phillips, Chia Shen, Morrill Hall, N Ingalls Bldg, Ann Arbor, Zeina Atrash Leong, Florian Block, Judy Diamond, E. Margaret Evans, Brenda Phillips, and Chia Shen. 2012. Of BATs and APEs: an interactive tabletop game for natural history museums. Proceedings of the ACM SIGCHI Conference on Human Factors in Computing Systems, ACM Press, 2059-2068. http://doi.org/10.1145/2207676.2208355

27. Eva Hornecker. 2008. “I don’t understand it either, but it is cool"-visitor interactions with a multi-touch table in a museum. Proceedings of IEEE International Workshop on Horizontal Interactive Human Computer Systems, 113-120.

28. Thomas J. Humphrey and Joshua P. Gutwill (eds.). 2005. Fostering active prolonged engagement: The art of creating APE exhibits. Left Coast Press, San Francisco, CA, USA.

29. Junko Ichino, Kazuo Isoda, Ayako Hanai, and Tetsuya Ueda. 2013. Effects of the display angle in museums on user's cognition, behavior, and subjective responses. Proceedings of the ACM SIGCHI Conference on Human Factors in Computing Systems, ACM Press, 2979-2988. http://doi.org/10.1145/2470654.2481413

30. Philip I. S. Lei and Angus K. Y. Wong. 2009. The multipletouch user interface revolution. IT Professional 11, 1, 42-49. http://doi.org/10.1109/MITP.2009.19

31. Jun Liu, David Pinelle, Samer Sallam, Sriram Subramanian, and Carl Gutwin. 2006. TNT: Improved Rotation and Translation on Digital Tables. Proceedings of Graphics Interface, Canadian Information Processing Society, 25-32.

32. Paul Marshall, Richard Morris, Yvonne Rogers, Stefan Kreitmayer, and Matt Davies. 2011. Rethinking “multi-user”: an in-the-wild study of how groups approach a walk-up-anduse tabletop interface. Proceedings of the ACM SIGCHI Conference on Human Factors in Computing Systems, ACM Press, 3033-3042. http://doi.org/10.1145/1978942.1979392

33. Lorna McKnight and Brendan Cassidy. 2010. Children's Interaction with Mobile Touch-Screen Devices. International Journal of Mobile Human Computer Interaction 2, 2, 18 pp. http://doi.org/10.4018/jmhci.2010040101

34. Lorna Mcknight and Daniel Fitton. 2010. Touch-screen Technology for Children : Giving the Right Instructions and Getting the Right Responses. Proceedings of the International Conference on Interaction Design and Children, ACM Press, 238-241. http://doi.org/10.1145/1810543.1810580

35. Ian Christopher McManus. 2009. The history and geography of human handedness. In Language lateralization and psychosis (1st ed.), Iris E. C. Sommer and René S. Kahn (eds.). Cambridge University Press, 37-58.

36. Paulette M. McManus. 1988. Good companions: More on the social determination of learning-related behaviour in a science museum. International Journal of Museum Management and Curatorship 7, 1, 37-44. http://doi.org/10.1080/09647778809515102

37. Meredith Ringel Morris, Jacob O. Wobbrock, and Andrew D. Wilson. 2010. Understanding users' preferences for surface gestures. Proceedings of Graphics Interface, Canadian Information Processing Society, 261-268.

38. Peter Peltonen, Esko Kurvinen, Antti Salovaara, Giulio 
Jacucci, Tommi Ilmonen, John Evans, Antti Oulasvirta, and Petri Saarikko. 2008. It's Mine, Don't Touch!: interactions at a large multi-touch display in a city centre. Proceeding of the ACM SIGCHI Conference on Human Factors in Computing Systems, ACM Press, 1285-1294. http://doi.org/10.1145/1357054.1357255

39. Molly Phipps. 2010. Research Trends and Findings From a Decade (1997-2007) of Research on Informal Science Education and Free-Choice Science Learning. Visitor Studies 13, 1, 3-22. http://doi.org/10.1080/10645571003618717

40. J.A. Pickering. 1986. Touch-sensitive screens: the technologies and their application. International Journal of Man-Machine Studies 25, 3, 249-269. http://doi.org/10.1016/S0020-7373(86)80060-8

41. Anne Marie Piper and James D. Hollan. 2009. Tabletop displays for small group study. Proceedings of the ACM SIGCHI Conference on Human Factors in Computing Systems, ACM Press, 1227-1236. http://doi.org/10.1145/1518701.1518885

42. Jochen Rick, Amanda Harris, Paul Marshall, Rowanne Fleck, Nicola Yuill, and Yvonne Rogers. 2009. Children designing together on a multi-touch tabletop: An analysis of spatial orientation and user interactions. Proceedings of the International Conference on Interaction Design and Children, ACM Press, 106-114. http://doi.org/10.1145/1551788.1551807

43. Yvonne Rogers and Siân Lindley. 2004. Collaborating around vertical and horizontal large interactive displays: which way is best? Interacting with Computers 16, 6, 1133-1152. http://doi.org/10.1016/j.intcom.2004.07.008

44. Jeremy M. Roschelle, Roy D. Pea, Christopher M. Hoadley, Douglas N. Gordin, and Barbara Means. 1999. Changing How and What Children Learn in School with Computer-based Technologies. Future of Children 10, 2, 76-101.

45. Karen Rust, Meethu Malu, Lisa Anthony, and Leah Findlater. 2014. Understanding child-defined gestures and children's mental models for touchscreen tabletop interaction. Proceedings of the International Conference on Interaction Design and Children, ACM Press, 201-204. http://doi.org/10.1145/2593968.2610452

46. Kathy Ryall, Meredith Ringel Morris, Katherine Everitt, Clifton Forlines, and Chia Shen. 2006. Experiences with and observations of direct-touch tabletops. Proceedings of the IEEE International Workshop on Horizontal Interactive Human-Computer Systems, IEEE, 8pp. http://doi.org/10.1109/TABLETOP.2006.12

47. Bertrand Schneider, Megan Strait, Laurence Muller, Sarah Elfenbein, Orit Shaer, and Chia Shen. 2012. Phylo-Genie: engaging students in collaborative "tree-thinking" through tabletop techniques. Proceedings of the ACM SIGCHI Conference on Human Factors in Computing Systems, ACM Press, 3071-3080. http://doi.org/10.1145/2207676.2208720

48. Deborah F. Schwartz. 2005. Dude, Where's My Museum? Inviting Teens to Transform Museums. Retrieved January 24, 2016 from http://www.mercermuseum.org/wpcontent/uploads/2014/10/Dude-Wheres-My-Museum..pdf

49. Stacey D. Scott, M. Sheelagh, T. Carpendale, and Kori M. Inkpen. 2004. Territoriality in collaborative tabletop workspaces. Proceedings of the ACM Conference on Computer Supported Cooperative Work, ACM Press, 294 303. http://doi.org/10.1145/1031607.1031655

50. Orit Shaer, Megan Strait, Consuelo Valdes, Taili Feng, Michael Lintz, and Heidi Wang. 2011. Enhancing genomic learning through tabletop interaction. Proceedings of the ACM SIGCHI Conference on Human Factors in Computing Systems, ACM Press, 2817-2826. http://doi.org/10.1145/1978942.1979361

51. John C. Tang. 1991. Findings from observational studies of collaborative work. International Journal of Man-Machine Studies 34, 2, 143-160. http://doi.org/10.1016/00207373(91)90039-A

52. Radu-Daniel Vatavu, Gabriel Cramariuc, and Doina Maria Schipor. 2015. Touch interaction for children aged 3 to 6 years: Experimental findings and relationship to motor skills. International Journal of Human-Computer Studies 74, 54-76. http://doi.org/10.1016/j.ijhcs.2014.10.007

53. Andrew D. Wilson, Shahram Izadi, Otmar Hilliges, Armando Garcia-Mendoza, and David Kirk. 2008. Bringing physics to the surface. Proceedings of the ACM Symposium on User Interface Software and Technology, ACM Press, 67-76. http://doi.org/10.1145/1449715.1449728

54. Jacob O. Wobbrock, Htet Htet Aung, Brandon Rothrock, and Brad A. Myers. 2005. Maximizing the guessability of symbolic input. Extended Abstracts of the ACM SIGCHI Conference on Human Factors in Computing Systems, ACM Press, 1869-1872. http://doi.org/10.1145/1056808.1057043

55. Jacob O. Wobbrock, Meredith Ringel Morris, and Andrew D. Wilson. 2009. User-defined gestures for surface computing. Proceedings of the ACM SIGCHI Conference on Human Factors in Computing Systems, ACM Press, 1083-1092. http://doi.org/10.1145/1518701.1518866

56. Mike Wu and Ravin Balakrishnan. 2003. Multi-finger and whole hand gestural interaction techniques for multi-user tabletop displays. Proceedings of the ACM Symposium on User Interface Software and Technology, 193-202. http://doi.org/10.1145/964696.964718 